\title{
Values of Sinogram Affirmed Iterative Reconstruction Algorithm-Based Low-Dose Computed Tomography Imaging in Clinical Diagnosis of Cerebral Hemorrhage
}

\author{
Yifei Wang $\mathbb{D},{ }^{1}$ Ying Chen $\left(\mathbb{D},{ }^{2}\right.$ Jincai Fang $\mathbb{D}^{1},{ }^{1}$ Yunnong Song $\mathbb{D}^{1},{ }^{1}$ Jianguo Shen $\left(\mathbb{D},{ }^{1}\right.$ \\ and Jianchao Wang ${ }^{1}$ \\ ${ }^{1}$ Department of Neurosurgery, The Second Affiliated Hospital of Jiaxing University, Jiaxing, Zhejiang 314000, China \\ ${ }^{2}$ Department of Operating Nursing, The Second Affiliated Hospital of Jiaxing University, Jiaxing, Zhejiang 314000, China \\ Correspondence should be addressed to Ying Chen; 141002310208@st.sdju.edu.cn
}

Received 28 August 2021; Revised 29 September 2021; Accepted 1 October 2021; Published 21 October 2021

Academic Editor: Gustavo Ramirez

Copyright (c) 2021 Yifei Wang et al. This is an open access article distributed under the Creative Commons Attribution License, which permits unrestricted use, distribution, and reproduction in any medium, provided the original work is properly cited.

\begin{abstract}
In this study, the sinogram affirmed iterative reconstruction (SAFIRE) technology combined with low-dose computed tomography (CT) scanning technology was applied to scan patients with cerebral hemorrhage to evaluate the application value of this combined technology in the diagnosis of patients with cerebral hemorrhage. 132 patients with cerebral hemorrhage admitted to the hospital were selected randomly as the research objects in this study. According to the patients' wishes, all of them were rolled into an experimental group and a control group. Besides, patients who were willing to use low-dose CT imaging scanning technology based on the SAFIRE algorithm were classified into the experimental group, and those who received simple low-dose CT scans were classified into the control group. Subjective score, objective score (mean CT value), image noise (SD), signal-to-noise ratio (SNR), and contrast-noise ratio (CNR) were used to analyze and compare the two groups of detection methods. The results showed the following: (1) The average result of CT images was $4.05 \pm 0.28$ points for the control group and $4.43 \pm 0.59$ points for the experimental group. According to the classification, the excellent and good rates of the experimental group (89.9\%) were markedly higher than the rates of the control group $(65.8 \%)$, with a statistically obvious difference $(P<0.05)$. (2) The standard deviation (SD), image signal-to-noise ratio (SNR), and contrast-noise ratio (CNR) results of gray matter, white matter, and cerebral hemorrhage lesions of patients from the experimental group were better than those of the control group, and the differences were statistically significant $(P<0.05)$. In conclusion, the image quality of a low-dose CT scan reconstructed by the SAFIRE algorithm was superior to that of a simple low-dose CT scan. In other words, the image quality of CT images could be guaranteed by the SAFIRE technology under the condition of reducing radiation dose. It enabled patients with cerebral hemorrhage to carry out diagnostic examinations with guaranteed accuracy under the risk of low radiation and provided better imaging services for patients with cerebral hemorrhage.
\end{abstract}

\section{Introduction}

Cerebral hemorrhage is a cerebrovascular disease that refers to the bleeding caused by the rupture of the blood vessels of the nontraumatic brain parenchymal tissue, which is a more common clinical disease [1]. The main cause of cerebral hemorrhage is the pathology of cerebrovascular tissue, which is closely related to factors such as hyperlipidemia, diabetes, high blood pressure, aging of blood vessels, and smoking [2]. Patients suffering from intracerebral hemorrhage often take emotional excitement or excessive exertion as the inducement, and there is a very high mortality rate in the early stage of the disease, while most of those who survive will have sequelae to varying degrees $[3,4]$. This disease accounts for $20 \%-30 \%$ of all stroke patients and is characterized by rapid onset, with the mortality rate of patients in the acute stage reaching 30\%-40\% [5,6]. Therefore, accurate and timely clinical diagnosis is essential. At the present stage, the main means of examination for suspected patients with intracerebral hemorrhage is a craniocerebral computed tomography (CT) scan. However, as the condition of intracerebral hemorrhage is prone to recurrence and changes, 
multiple scans of intracerebral hemorrhage patients are needed to determine the condition of the disease, so that the clinical treatment plan can be changed [7,8]. The images scanned by CT with the features of high resolution, clear pixels, and no artifacts can provide clear diagnostic evidence for the clinical treatment of patients. However, studies have found that plain CT examination is a means of examination with the highest radiation dose, and multiple CT scans will lead to an increase in radiation dose, which will have adverse effects on the health of patients $[9,10]$. Therefore, the dose of scanning ray should be reduced as far as possible while not affecting the diagnosis of a cerebral hemorrhage.

The sinogram affirmed iterative reconstruction (SAFIRE) algorithm is a new iterative reconstruction algorithm developed by Siemens, which is an iterative reconstruction algorithm based on the original data [11]. It can extremely reduce the noises of CT images and has a significant effect on the removal of spiral CT artifacts. This algorithm can be applied in CT image reconstruction to effectively improve the image quality, which can further reduce the radiation dose of CT scans [12]. Moreover, related studies have proved that the improvement of SAFIRE technology can decrease the CT scan dose without increasing the noise of the CT image, and the impact on the image quality is relatively small [13].

Therefore, the SAFIRE algorithm was combined with low-dose CT scanning technology in this study to greatly reduce the amount of harmful radiation during the CT scan of patients with cerebral hemorrhage and to normally show the scanned CT images of the patients' condition. In addition, the method was adopted in the examination and diagnosis of the disease of patients with cerebral hemorrhage, to evaluate the diagnostic value of the method, thereby providing a more advantageous and less harmful examination method for the diagnosis and treatment of the disease of patients with cerebral hemorrhage.

\section{Research Methods}

2.1. Research Objects. In this study, 132 patients with cerebral hemorrhage were selected randomly as the research objects, who were admitted to the hospital from May 2018 to March 2021. Among them, 79 were male patients and 53 were female patients; they were 35-75 years old, with an average age of $56.67 \pm 3.31$ years old. Then, all patients were divided into the experimental group and the control group based on their wishes. 59 patients who were willing to be examined by the low-dose CT imaging technology based on the SAFIRE algorithm were assigned to the experimental group. Other patients who did not want to use the combination method were examined using conventional low-dose CT scans and assigned to a control group of 73 patients. In addition, this study was approved by the Medical Ethics Committee.

\subsection{CT Examination and SAFIRE Technology Reconstruction}

2.2.1. CT Examination. All patients were examined by lowdose CT scanning, and the scanning equipment used was Siemens Dual-Source Flash CT (SOMATOM definition Flash, Siemens Healthcare, Forchheim, Germany).
Scanning position of the patients: they took the supine position, the head was placed on the head frame, the mandible was retracted, the sagittal plane of the skull and body coincided with the midline of the mesa, the two external ear holes were equidistant from the mesa, the hands were placed on both sides of the body, and the scanning baseline was the auditory canthus line. Besides, the specific scanning method is shown in Figure 1.

2.2.2. SAFIRE Technology Reconstruction. The CT images of the patients in the experimental group were reconstructed using the SAFIRE algorithm. The brain CT reconstruction parameters of the SAFIRE iterative algorithm were SAFIRE 3 , and the convolution kernel J30s medium smooth. The conventional CT images of the control group were used as the scanning results of the examination. SAFIRE algorithm introduced two sets of cyclic iteration based on the initial iterative reconstruction technology.

First, the iterative process in the image domain: the original data are used to reconstruct the image, and the image prior knowledge is used to perform repeated iterative correction in the image space. This process is image denoising, which does not reduce the contrast of the image. It is assumed that the initial image is $X$; then, $\Delta X$ updates a correction value each time.

$$
\Delta X=\left\{\begin{array}{l}
\Delta X_{k}, \quad\left(k \leq q_{i} \leq 0\right), \\
\Delta X_{1},
\end{array}\right.
$$

where $k$ represents the iteration sequence number and $q_{i}$ represents the real number.

Second, the result of image domain iteration is transformed into the original data domain. A CT simulator is established in the computer, the result of image domain iteration is used as the inspection object, and the simulated scan result is obtained through calculation. The difference between the simulated scan results and the actual original data is used as the noise template, which is converted to the imaging domain by FBP and superimposed on the last reconstructed image, and the iterative reconstruction of the image domain is carried out again. This cycle is repeated 1.5 times to obtain the final diagnostic image.

\subsection{Evaluation Indicators}

(1) CT images of patients from the two groups were scored subjectively according to the scoring criteria [14], as shown in Table 1. The main method was to average the scores of two clinically experienced chief physicians (more than 20 years of working experience) based on the gray-white matter boundary, sulcus, cistern display, image sharpness, and diagnostic acceptability. Moreover, there were 5 grades, including unqualified (1-2 points), qualified (3 points), good (4 points), and excellent (5 points); the grading was carried out based on the above. Furthermore, the excellent and good rate $=($ the number of the excellent + the number of the good)/total cases $\times 100 \%$. 


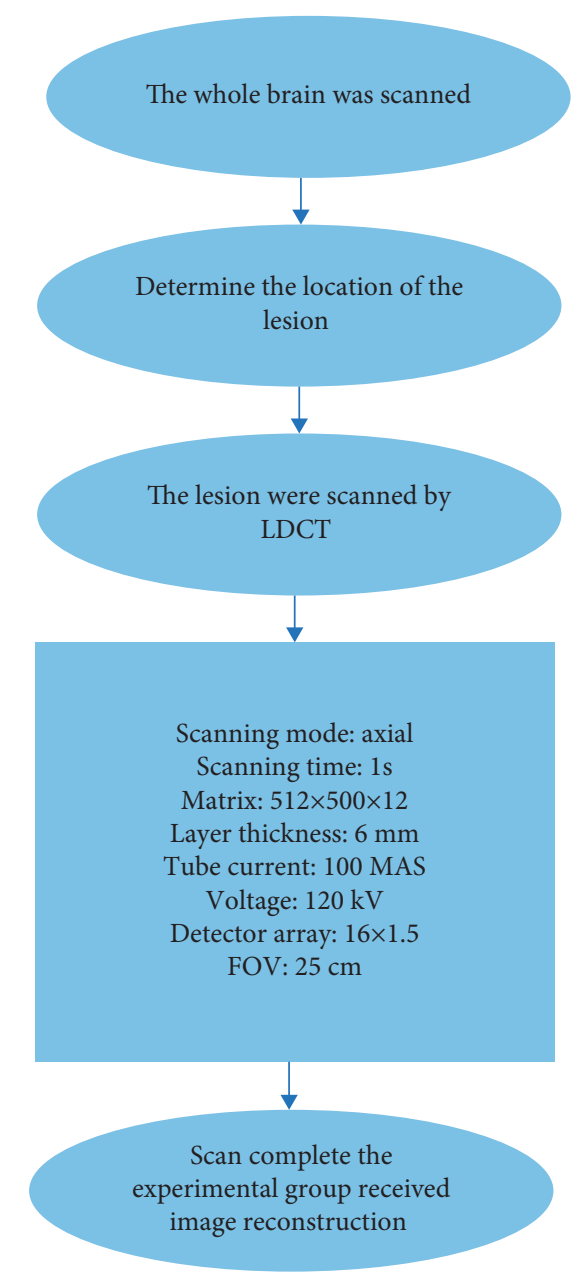

Figure 1: The specific operation process of CT scan.

(2) CT images of patients from the two groups were objectively scored, including the average CT values of gray matter, white matter, cerebral hemorrhage, standard deviation (SD) of the image noise, signal-to-noise ratio (SNR), and contrastnoise ratio (CNR). Average $\mathrm{CT}$ values were measured at the body level of the lateral ventricle, and the region of interest (ROI) was set at $5 \mathrm{~mm}^{2}$. Cerebral sulci and blood vessels were avoided as far as possible during measurement. Image noise refers to the standard deviation (SD) of average $\mathrm{CT}$ value. Then, the expression equation of SNR and CNR is as follows:

$$
\begin{aligned}
\mathrm{SNR} & =\frac{\mathrm{CT}_{P}}{\mathrm{SD}}, \\
\mathrm{CNR} & =\frac{\mathrm{CT}_{P_{l}}-\mathrm{CT}_{P_{\prime \prime}}}{\overline{\mathrm{SD}}},
\end{aligned}
$$

where $\mathrm{CT}_{P}$ is the average $\mathrm{CT}$ value, $\mathrm{CT}_{P}$, is the average CT value of cerebral hemorrhage focus, $C T_{P_{\| \prime}}$ is the average CT value of brain parenchyma, and $\mathrm{SD}$ is the standard deviation of background SD [15].
2.4. Statistical Methods. The SPSS22.0 statistical software system was used for data entry, sorting, and statistical analysis. The count data were compared by $\mathrm{X}^{2}$ test, and the measurement data were compared by the $t$-test. Analysis of variance (ANOVA) was used for comparison of multiple sample average values, the least significant difference (LSD) method was used for homogeneity of variances, and the Dunnett's T3 method was used for heterogeneity of variances. Besides, $P<0.05$ indicated that the difference was statistically substantial. The Kappa test was conducted for the consistency of subjective scores of the two physicians. When Kappa $>0.75$, the consistency was strong; when $0.4 \leq$ Kappa $<0.75$, the consistency was moderate; and when Kappa $<0.4$, the consistency between the two was poor.

\section{Results}

3.1. Comparison of General Treatment. The statistics of age, sex, bleeding location, causes of intracerebral hemorrhage, and other general data of patients in the experimental group and the control group was performed (Table 2). In terms of gender distribution, the proportion of male patients was $60.27 \%(44 / 73)$ in the control group and $59.32 \%(35 / 59)$ in the experimental group. The proportion of female patients was $39.73 \%(29 / 73)$ in the control group and $40.68 \%(24 / 59)$ in the experimental group. In terms of average age, the control group was $(54.67 \pm 2.89)$ years old. The experimental group was $(56.01 \pm 3.22)$ years old. As for the bleeding site, putamen was $46.58 \%$ (34/73) in the control group and $47.45 \%(28 / 59)$ in the experimental group. Cerebral cortex was $24.66 \%(18 / 73)$ in the control group and $27.12 \%(16 / 59)$ in the experimental group. Cerebellum was $15.07 \%(11 / 73)$ in the control group and $11.86 \%(7 / 59)$ in the experimental group. Thalamus was $9.59 \%$ (7/73) in the control group and $8.47 \%(5 / 59)$ in the experimental group. Simple ventricle was $4.11 \%(3 / 73)$ in the control group and $5.08 \%(3 / 59)$ in the experimental group. As for the causes of bleeding, traumatic bleeding was $43.84 \%(32 / 73)$ in the control group and $45.76 \%(27 / 59)$ in the experimental group. Hypertensive hemorrhage was $39.73 \%(29 / 73)$ in the control group and $38.98 \%(23 / 59)$ in the experimental group. Cerebral vascular malformation caused hemorrhage: $16.44 \%(12 / 73)$ in the control group and $15.25 \%(9 / 59)$ in the experimental group. The comparison of the above statistical results is not statistically significant $(P>0.05)$, suggesting that the research results of the above two groups are comparable.

3.2. CT Imaging Manifestations of Different Parts of the Cerebral Hemorrhage in Patients from the Control Group. Figure 2 shows the CT image performance of different hemorrhage sites of different patients with cerebral hemorrhage in the control group during this study. Figure 2(a) shows a CT image of a patient with hemorrhage in the cerebellum; Figure 2(b) shows a CT image of a patient with hemorrhage in the putamen; Figure 2(c) shows a CT image of a patient with hemorrhage in the hypothalamus; Figure 2(d) shows a CT image of a patient with hemorrhage in the ventricle. In the CT images of the above different 
TABLE 1: Subjective scores and grading standards of the CT images.

\begin{tabular}{lcc}
\hline Grade & $\begin{array}{c}\text { Score } \\
\text { (points) }\end{array}$ & Display standard \\
\hline $\begin{array}{l}\text { Disqualification } \\
\text { Qualification }\end{array}$ & 1 & The anatomical structure and lesion of the image were blurred and there were many artifacts, so it could \\
not be completely diagnosed. \\
Good
\end{tabular}

TAвLE 2: General clinical data statistics of the two groups of patients.

\begin{tabular}{|c|c|c|c|}
\hline & & Experimental group $(n=59)$ & Control group $(n=73)$ \\
\hline \multirow{2}{*}{ Gender } & Female & $35(59.32 \%)$ & $44(60.27 \%)$ \\
\hline & Male & $24(40.68 \%)$ & $29(39.73 \%)$ \\
\hline \multirow{3}{*}{ Average age (years) } & & $54.67 \pm 2.89$ & $56.01 \pm 3.22$ \\
\hline & Putamen & $28(47.45 \%)$ & $34(46.58 \%)$ \\
\hline & Cerebral hemispheric cortex & $16(27.12 \%)$ & $18(24.66 \%)$ \\
\hline \multirow[t]{4}{*}{ Bleeding site } & Cerebellum & $7(11.86 \%)$ & $11(15.07 \%)$ \\
\hline & Thalamus & $5(8.47 \%)$ & $7(9.59 \%)$ \\
\hline & Pure ventricle & $3(5.08 \%)$ & $3(4.11 \%)$ \\
\hline & Traumatic & $27(45.76 \%)$ & $32(43.84 \%)$ \\
\hline \multirow[t]{2}{*}{ Cause of cerebral hemorrhage } & Hypertensive & $23(38.98 \%)$ & $29(39.73 \%)$ \\
\hline & Cerebrovascular malformation & $9(15.25 \%)$ & $12(16.44 \%)$ \\
\hline
\end{tabular}

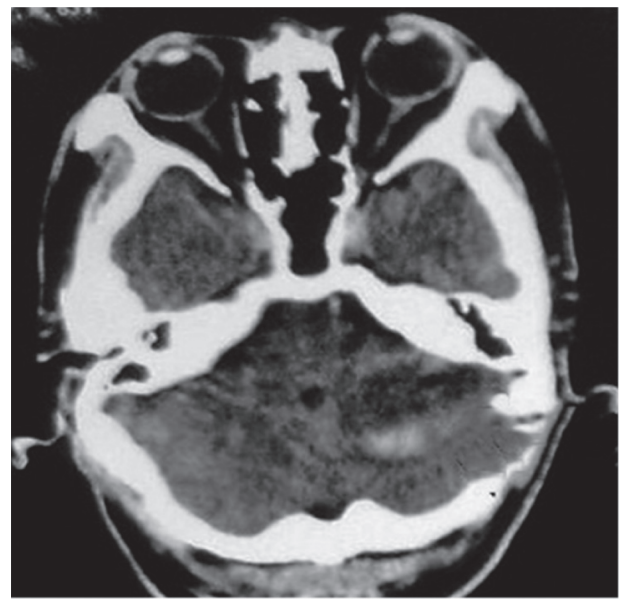

(a)

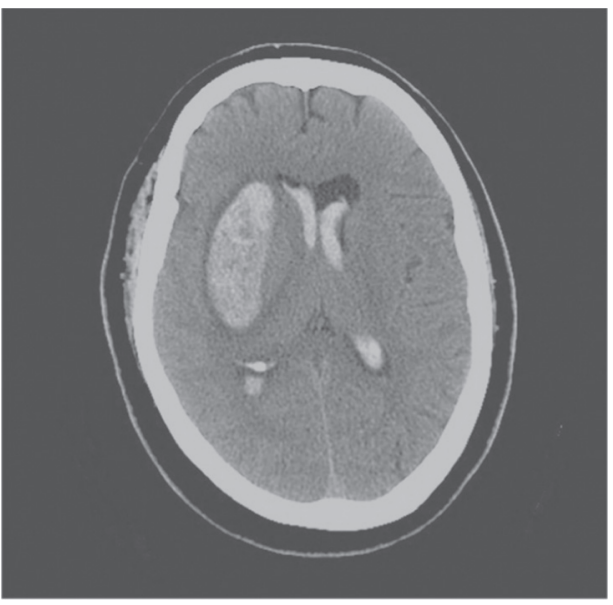

(b)

Figure 2: Continued. 


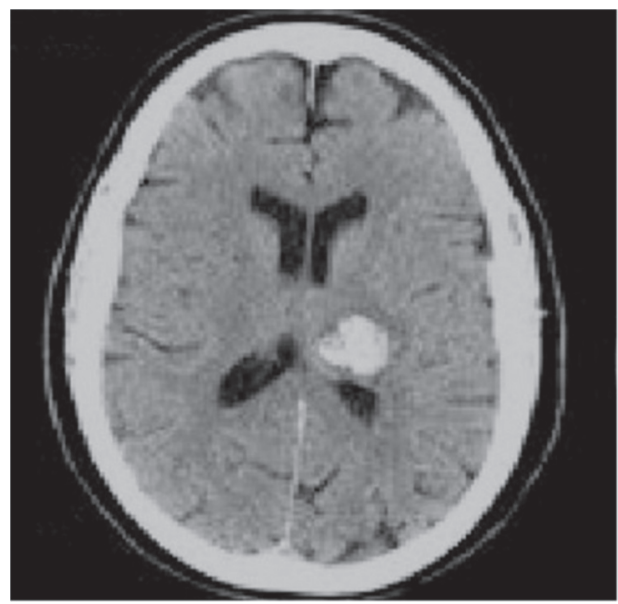

(c)

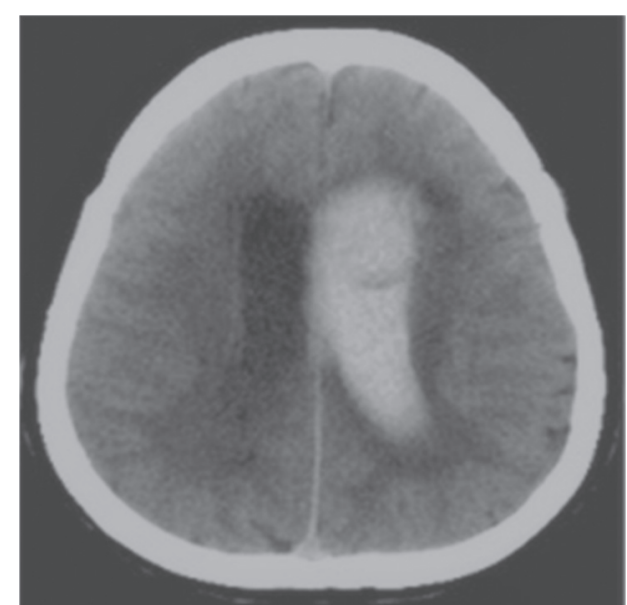

(d)

FIGURE 2: CT images of different parts of the cerebral hemorrhage in patients from the control group. (a) Cerebellum, male, 56 years old. (b) Putamen, male, 55 years old. (c) Thalamus, female, 46 years old. (d) Ventricle, female, 48 years old.

bleeding sites, bleeding high-signal shadows of different areas and sizes could be observed, but the display was not very clear and the resolution was not high. The signal display between the normal brain tissue was poor, and the boundary line was not clear.

3.3. CT Imaging Manifestations of Different Parts of the Cerebral Hemorrhage in Patients from the Experimental Group. The CT images of different sites of the cerebral hemorrhage in different patients from the experimental group during this study are shown in Figure 3. Besides, Figure 3(a) shows a CT image of one patient with cerebellar hemorrhage; Figure 3(b) shows a CT image of one patient with putamen hemorrhage; Figure 3(c) shows a CT image of one patient with hypothalamic hemorrhage; Figure $3(\mathrm{~d})$ shows a CT image of one patient with ventricular hemorrhage. Through the observation of CT images of patients in the experimental group, it was found that the high and low signal display of this group of images was very obvious, the lesion site was also very clear, and the boundary between the lesion and the normal tissue was clear.

\subsection{Subjective Scores and Grading of CT Images of the Cerebral} Hemorrhage in the Two Groups. By sorting and counting the subjective score and grading of CT images of patients in the two groups by two physicians (Table 3), the score of the control group by physician A was $4.01 \pm 0.23$ points, and the score by physician B was $4.09 \pm 0.33$ points. The score results of the experimental group by physicians $A$ and $B$ were $4.34 \pm 0.56$ points and $4.52 \pm 0.61$ points in turn. There was no statistical significance in the comparison between the data in the two groups $(P>0.05)$ (Figure 4). After the Kappa test, the CT image quality scores of patients from the two groups by two physicians were consistent (Kappa value was 0.75 and 0.78 , respectively), and there was no statistically marked difference in this comparison $(P>0.05)$. The average CT score of the two groups was $4.05 \pm 0.28$ points in the control group and $4.43 \pm 0.59$ points in the experimental group. There were 45 cases rated as excellent and 8 cases rated as good in the experimental group, so the excellent and good rate was $89.9 \%$ (53/59). In the control group, 38 cases were excellent and 10 cases were good, so the excellent and good rate was $65.8 \%$ (48/73). Through comparison, it was found that the excellent and good rate of grading in the experimental group was significantly higher than that in the control group $(P<0.05)$, and the quality of CT images in the experimental group was higher than that in the control group (Figure 5), suggesting that the diagnosticity of CT images reconstructed by SAFIRE algorithm was higher.

3.5. CT Imaging Manifestations and Objective Scores of the Cerebral Hemorrhage in Patients from the Two Groups. By sorting out and comparing the objective scores of the CT images of the two groups of patients, it was found that there were certain differences in the average CT values of the gray matter, white matter, and cerebral hemorrhage lesions of the two groups of CT images, but there was no marked difference $(P<0.05)$ (Figure 6). However, the SD, image SNR, and CNR results of gray matter, white matter, and cerebral hemorrhage lesions in the experimental group were better than those in the control group, and the differences were statistically significant $(P<0.05)$ (Figure 7$)$.

\section{Discussion}

Cerebral hemorrhage, a common sudden disease in clinical practice, has the highest mortality rate in acute cerebrovascular diseases, and due to the frequent and recurrent nature of this disease, it is necessary to carry out CT examination repeatedly for the diagnosis and treatment of the disease [16]. Moreover, the diagnostic effect of CT on cerebral hemorrhage disorders has been investigated by many experts, and studies have concluded that CT can not only display the location range, size, and the number of lesions of 


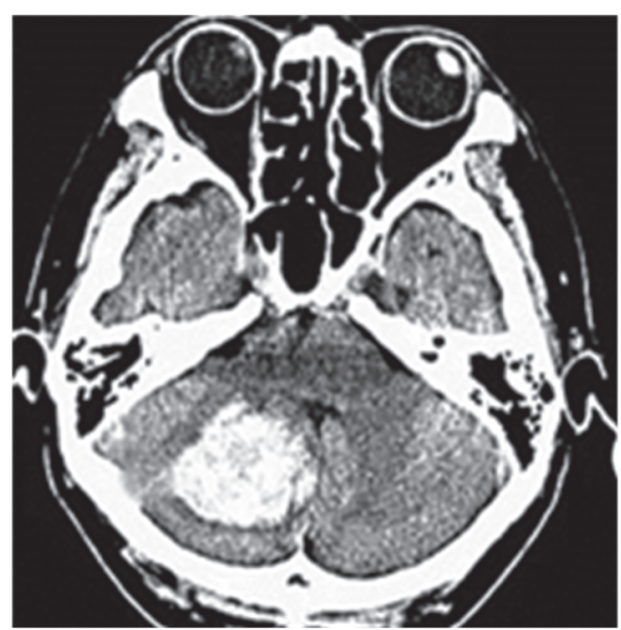

(a)

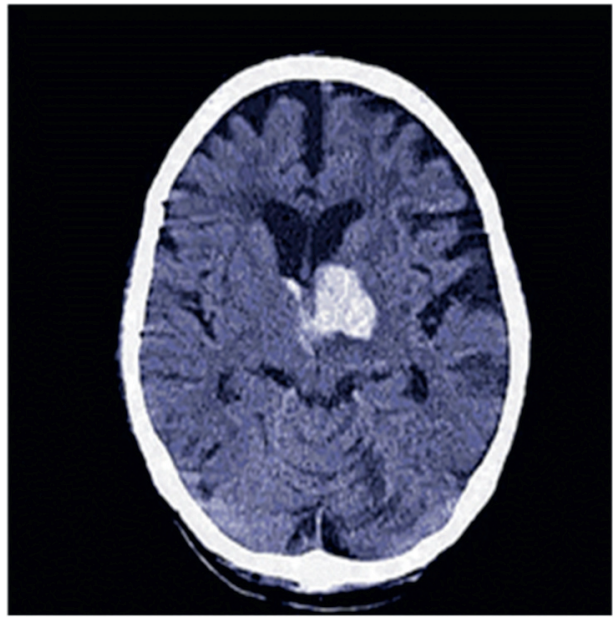

(c)

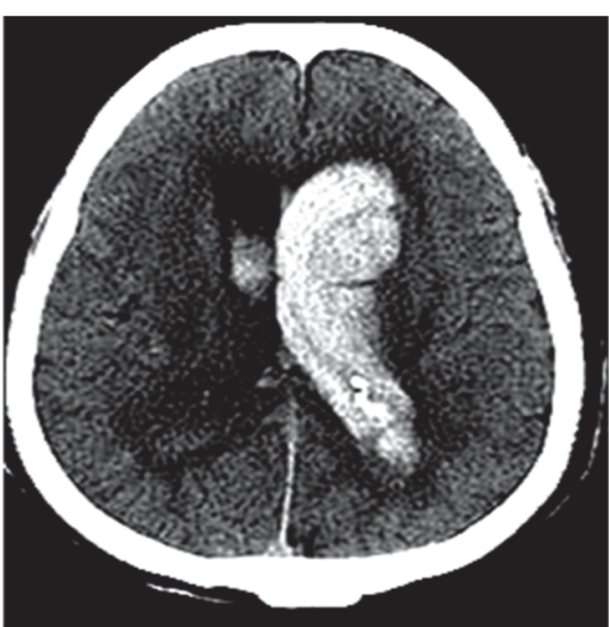

(b)

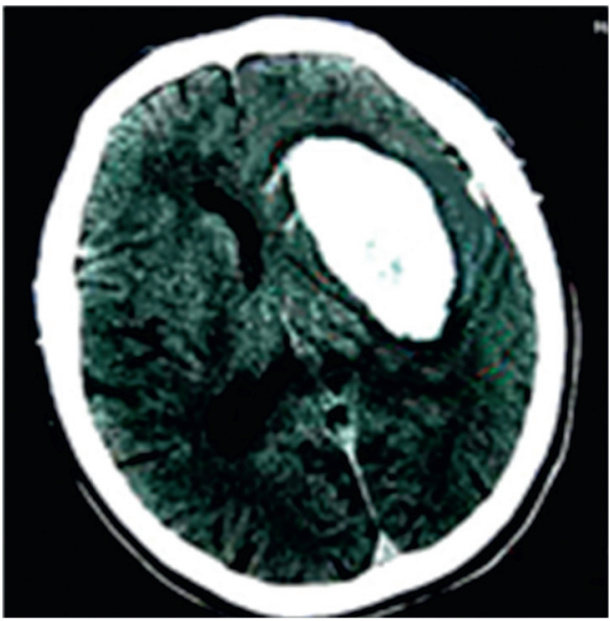

(d)

FIGURE 3: CT imaging manifestations of different parts of the cerebral hemorrhage in the experimental group. (a) Cerebellum, female, 50 years old. (b) Putamen, male, 43 years old. (c) Thalamus, male, 54 years old. (d) Ventricle, female, 57 years old.

TABLE 3: Subjective scores and grading of CT images of patients from the two groups.

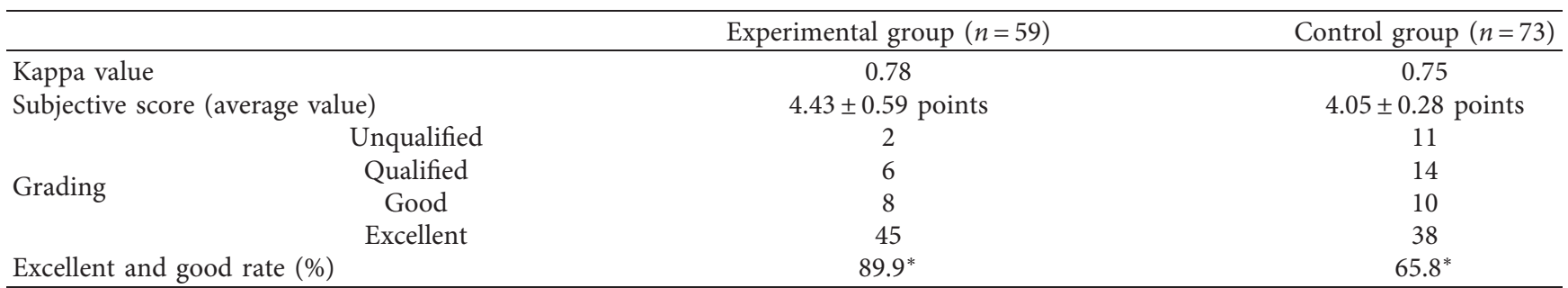

Note. ${ }^{*}$ The comparison is statistically significant $(P<0.05)$.

cerebral hemorrhage but also dynamically display lesions, providing an effective basis for clinical treatment of cerebral hemorrhage [17]. However, there is electrical radiation in the operation of CT inspection, and repeated CT scans in a short period have a great radiation risk, so the low-dose CT examination technology has become the focus of research in recent years $[18,19]$. Some experts have conducted relevant studies on the application of low-dose CT imaging technology in the examination of craniocerebral diseases, and the results reveal that low-dose CT scan can form a good contrast with the surrounding brain tissue, which provides a basis for the application of low-dose CT scan in the examination of cerebral hemorrhage [20]. Later, there have been some studies where experts have analyzed the 


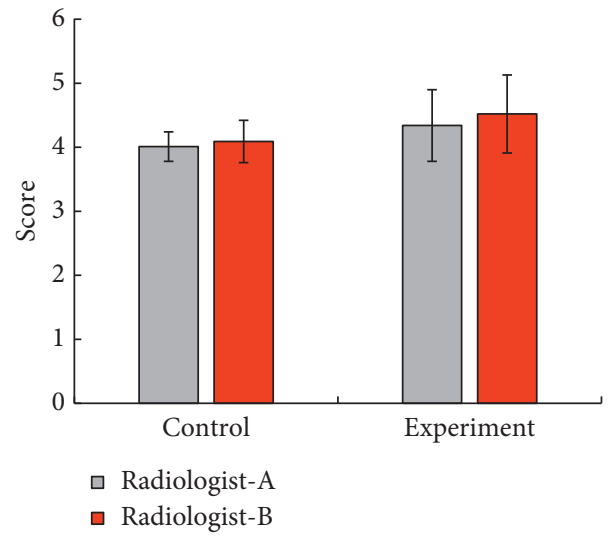

Figure 4: Comparison of the subjective scores from two physicians.

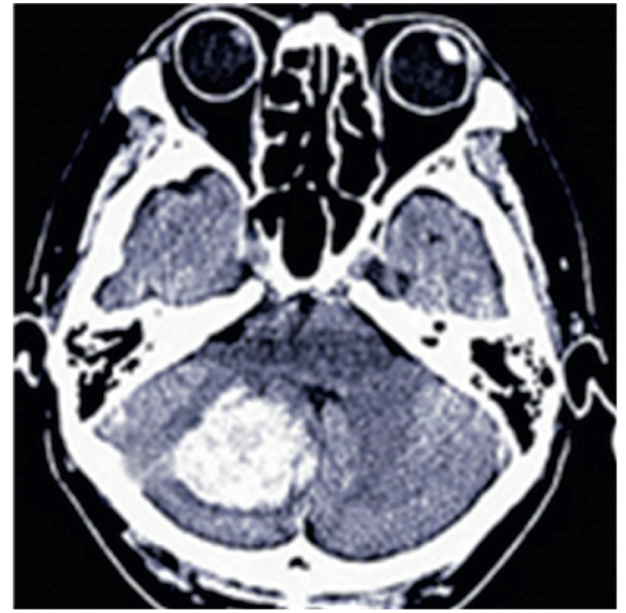

Experiment

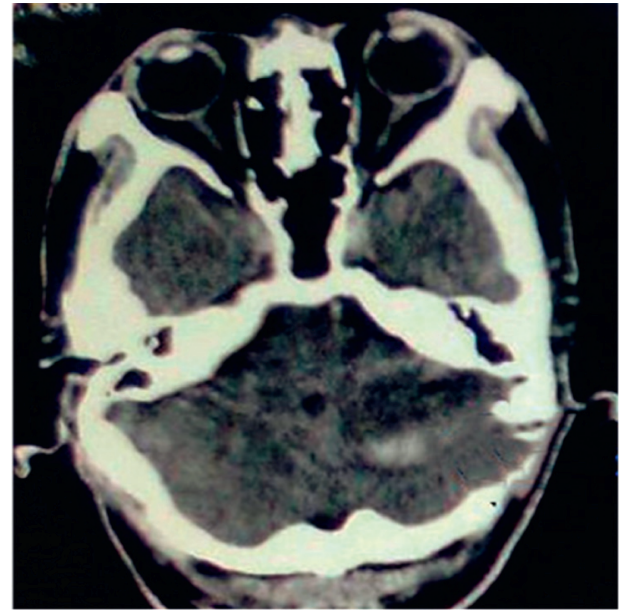

Control

FIGURE 5: Comparison of CT images of the two groups of patients. The figure shows the two CT images of the cerebellar area of two patients.

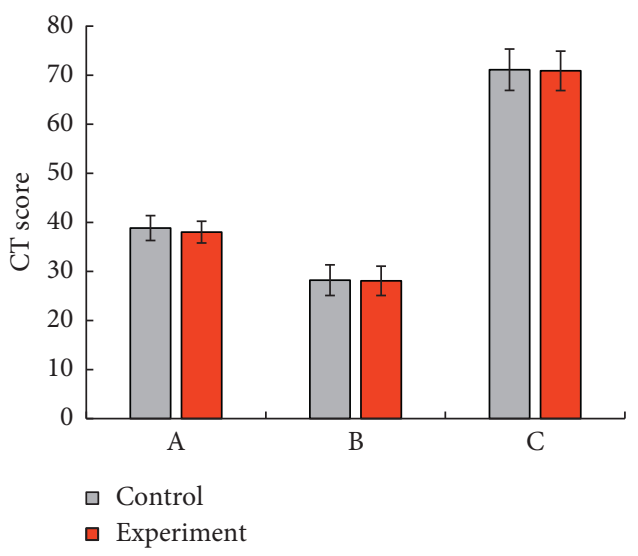

FIGURE 6: CT average of each organization. A: gray matter; B: white matter; C: hematoma.

application of low-dose CT scanning in the detection of a cerebral hemorrhage. The results indicate that the low-dose CT scan can achieve the same effect as routine dose CT scan in the examination of patients with cerebral hemorrhage and is conducive to reducing the radiation impact on patients, so

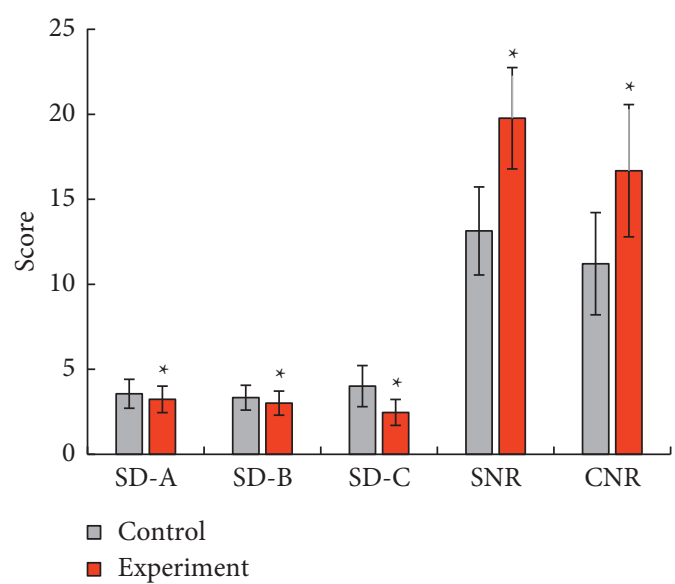

FIgURe 7: The objective scores for the image quality of the two groups. SD-A: SD of gray matter; SD-B: SD of white matter; SD-C: $\mathrm{SD}$ of hematoma. Note: * the comparison is statistically significant $(P<0.05)$

it has a good application value $[8,21]$. A recent meta-analysis has shown that, in the low-dose CT imaging of patients with atherosclerotic subarachnoid hemorrhage, if the radiation 
dose is reduced to $40 \%$ of the original dose level, the accuracy of the diagnosis of cerebral perfusion injury will not be affected [7]. However, some experts put forward that there is an exponential relationship between tube voltage and radiation dose, and reducing tube voltage can significantly reduce radiation dose, but at the same time increase image noise and reduce image quality $[13,22]$. Therefore, with the continuous improvement of multi-slice spiral CT technology, the SAFIR technology has been studied.

In this study, when low-dose CT scanning technology was used to examine patients with cerebral hemorrhage, SAFIR technology was employed to reconstruct CT images, and the results of routine low-dose CT scanning were compared for the diagnosis of the disease. The results disclosed that the average CT scores of patients in the two groups were $4.05 \pm 0.28$ points in the control group and $4.43 \pm 0.59$ points in the experimental group. The excellent and good rate of the experimental group was $89.9 \%(53 / 59)$, and the rate of the control group was $65.8 \%$ (48/73). Besides, the SD, image SNR, and CNR results of gray matter, white matter, and cerebral hemorrhage lesions in the experimental group were better than those in the control group, and the differences were statistically remarkable $(P<0.05)$, suggesting that low-dose CT images after reconstruction by SAFIR technology had more diagnostic significance. Some experts have conducted a preliminary study on the clinical application of low-tube voltage combined with iterative reconstruction technology in CT examination of patients with cerebral hemorrhage, and the results show that lowtube voltage combined with SAFIR level 3 reconstruction technology can effectively reduce radiation dose on the premise of ensuring image quality. Therefore, on the premise of ensuring diagnostic accuracy, the risk of ionizing radiation of subjects can be reduced and better imaging services can be provided for patients with cerebral hemorrhage [23]. Low-dose CT scanning combined with SAFIRE for the diagnosis of lumbar disc herniation (LDH) has been studied, indicating that the image quality of low-dose CT scanning combined with SAFIR LDH examination is no less than that of the routine dose; compared with the routine-dose scanning, low-dose CT scanning does not affect the diagnostic effect, and it is in good agreement with clinical diagnosis results, which also has ideal application value [24]. There is also research that evaluates the image quality of Filtered Back Projection (FBP) and SAFIRE in the context of low radiation and low-contrast CT scanning in aorta examination. The results have indicated that $70 \mathrm{kV}$ combined with SAFIR iterative reconstruction is feasible in the application of pulmonary artery CT angiography. SAFIR has lower noise than FBP and plays an important role in lowdose studies [25]. The above results are similar to the results of this study, showing the application advantages of SAFIR technology.

\section{Conclusion}

In this study, SAFIR technology combined with low-dose CT scanning technology was applied to scan patients with cerebral hemorrhage, and the image quality under the technology was compared with that under the low-dose CT scanning technology alone, to evaluate the application value of this combined technology in the diagnosis of cerebral hemorrhage. The results showed that the image quality of low-dose CT scan reconstructed by SAFIRE algorithm was better than that of low-dose CT scan, so that patients with cerebral hemorrhage could carry out diagnostic examination with guaranteed accuracy under the risk of low radiation and provide better imaging services for patients with cerebral hemorrhage. However, this research still has certain limitations, the comparison indicators are not perfect, and the research content is not very representative. Therefore, future research will be strengthened and improved. In addition, the application prospect of SAFIRE technology has been reflected to a certain extent.

\section{Data Availability}

The data used to support the findings of this study are available from the corresponding author upon request.

\section{Conflicts of Interest}

The authors declare no conflicts of interest.

\section{References}

[1] J. Peng, H. Wang, X. Rong et al., "Cerebral hemorrhage and alcohol exposure: a review," Alcohol and Alcoholism, vol. 55, no. 1, pp. 20-27, 2020.

[2] I. C. Hostettler, D. J. Seiffge, and D. J. Werring, "Intracerebral hemorrhage: an update on diagnosis and treatment," Expert Review of Neurotherapeutics, vol. 19, no. 7, pp. 679-694, 2019, PMID: 31188036.

[3] J. Pinho, A. S. Costa, J. M. Araújo, J. M. Amorim, and C. Ferreira, "Intracerebral hemorrhage outcome: a comprehensive update," Journal of the Neurological Sciences, vol. 398, pp. 54-66, 2019, Epub 2019 Jan 14. PMID: 30682522.

[4] L. Sansing, "Intracerebral hemorrhage," Seminars in Neurology, vol. 36, pp. 223-224, 2016, Epub 2016 May 23. PMID: 27214696.

[5] M. Schrag and H. Kirshner, "Management of intracerebral hemorrhage," Journal of the American College of Cardiology, vol. 75, no. 15, pp. 1819-1831, 2020 Apr 21.

[6] A. Charidimou, A. Morotti, and G. Boulouis, "Cumulative meta-analysis of intensive blood-pressure lowering in acute cerebral hemorrhage: quo vadis?" Journal of the Neurological Sciences, vol. 375, pp. 179-180, 2017, Epub 2017 Jan 14. PMID: 28320125.

[7] S. Afat, C. Brockmann, O. Nikoubashman et al., "Diagnostic accuracy of simulated low-dose perfusion CT to detect cerebral perfusion impairment after aneurysmal subarachnoid hemorrhage: a retrospective analysis," Radiology, vol. 287, no. 2, pp. 643-650, 2018, Epub 2018 Jan 8. PMID: 29309735.

[8] D. Wu, G. Wang, B. Bian, Z. Liu, and D. Li, "Benefits of lowdose CT scan of head for patients with intracranial hemorrhage," Dose-response: A Publication of International Hormesis Society, vol. 19, no. 1, 2020.

[9] Y. Xin, S. Shi, G. Yuan, Z. Miao, Y. Liu, and Y. Gu, “Application of CT imaging in the diagnosis of cerebral hemorrhage and cerebral infarction nerve damage," World Neurosurgery, vol. 138, pp. 714-722, 2020, PMID: 32545021. 
[10] D. Shi, D. Jin, W. Cai et al., "Serial low-dose quantitative CT perfusion for the evaluation of delayed cerebral ischaemia following aneurysmal subarachnoid haemorrhage," Clinical Radiology, vol. 75, no. 2, pp. 131-139, 2020, Epub 2019 Nov 4. PMID: 31699431.

[11] S. Choy, D. Parhar, K. Lian et al., "Comparison of image noise and image quality between full-dose abdominal computed tomography scans reconstructed with weighted filtered back projection and half-dose scans reconstructed with improved sinogram-affirmed iterative reconstruction (SAFIRE*)," $A b$ dominal Radiology, vol. 44, no. 1, pp. 355-361, 2019.

[12] M. Scharf, S. Brendel, K. Melzer et al., "Image quality, diagnostic accuracy, and potential for radiation dose reduction in thoracoabdominal CT, using Sinogram Affirmed Iterative Reconstruction (SAFIRE) technique in a longitudinal study," PLoS One, vol. 12, no. 7, PMID: 28678818; PMCID: PMC5498038, Article ID e0180302, 2017.

[13] S. B. Nam, D. W. Jeong, K. S. Choo et al., "Image quality of CT angiography in young children with congenital heart disease: a comparison between the sinogram-affirmed iterative reconstruction (SAFIRE) and advanced modelled iterative reconstruction (ADMIRE) algorithms," Clinical Radiology, vol. 72, no. 12, pp. 1060-1065, 2017, Epub 2017 Jul 29. PMID: 28764856.

[14] R. R. Price, L. Axel, T. Morgan et al., "Quality assurance methods and phantoms for magnetic resonance imaging: report of AAPM nuclear magnetic resonance Task Group No. 1," Medical Physics, vol. 17, no. 2, pp. 287-295, 1990.

[15] C. Ozdoba, J. Slotboom, G. Schroth et al., "Dose reduction in standard head CT: first results from a new scanner using iterative reconstruction and a new detector type in comparison with two previous generations of multi-slice CT," Clinical Neuroradiology, vol. 24, no. 1, pp. 23-28, 2014, Epub 2014 Jan 31. PMID: 24482000; PMCID: PMC3936131.

[16] A. Morotti, G. Boulouis, D. Dowlatshahi et al., "Standards for detecting, interpreting, and reporting noncontrast computed tomographic markers of intracerebral hemorrhage expansion," Annals of Neurology, vol. 86, no. 4, pp. 480-492, 2019, Epub 2019 Aug 24. PMID: 31364773.

[17] A. Morotti, G. Busto, A. Bernardoni, C. Tamborino, and E. Fainardi, "Association between perihematomal cerebral blood volume and intracerebral hemorrhage expansion: a computed tomography perfusion study," Annals of Neurology, vol. 85, no. 6, pp. 943-947, 2019, Epub 2019 Apr 3. PMID: 30864197.

[18] D. E. Amare and H. Dagne, "Knowledge and associated factors of medical students regarding radiation exposure from common diagnostic imaging procedures at the University of Gondar, Ethiopia," Ethiopian journal of health sciences, vol. 30, no. 4, pp. 589-598, 2020, PMCID: PMC8054455.

[19] M. Messerli, A.-L. Panadero, A. A. Giannopoulos et al., "Enhanced radiation exposure associated with anterior-posterior x-ray tube position in young women undergoing cardiac computed tomography," American Heart Journal, vol. 215, pp. 91-94, 2019, Epub 2019 May 28. PMID: 31295633.

[20] A. E. Othman, S. Afat, C. Brockmann et al., "Low-dose volume-perfusion CT of the brain: effects of radiation dose reduction on performance of perfusion CT algorithms," Clinical Neuroradiology, vol. 27, no. 3, pp. 311-318, 2017, Epub 2015 Dec 15. PMID: 26669592.

[21] H. Yabuuchi, T. Kamitani, K. Sagiyama et al., "Clinical application of radiation dose reduction for head and neck CT," European Journal of Radiology, vol. 107, pp. 209-215, 2018, Epub 2018 Aug 24. PMID: 30177405.
[22] A. Vaniqui, L. E. J. R. Schyns, I. P. Almeida, B. van der Heyden, M. Podesta, and F. Verhaegen, "The effect of different image reconstruction techniques on pre-clinical quantitative imaging and dual-energy CT," British Journal of Radiology, vol. 92, no. 1095, Epub 2018 Nov 7. PMID: 30394804; PMCID: PMC6541183, Article ID 20180447, 2019.

[23] A. Korn, B. Bender, M. Fenchel et al., "Sinogram affirmed iterative reconstruction in head CT: improvement of objective and subjective image quality with concomitant radiation dose reduction," European Journal of Radiology, vol. 82, no. 9, pp. 1431-1435, 2013, Epub 2013 Apr 12. PMID: 23587902.

[24] M. Xiao, M. Zhang, J. Liu, G. Zhou, and W. Xu, "Iterative reconstruction combined with low dose $\mathrm{CT}$ in diagnosis of lumbar intervertebral disc hernia," Chinese Journal of Medical Imaging Technology, vol. 290, no. 2, pp. 446-455, 2019, Epub 2018 Dec 4. PMID: 30615548.

[25] A. Örgel, G. Bier, F. Hennersdorf, H. Richter, U. Ernemann, and T.-K. Hauser, "Image quality of CT angiography of supraaortic arteries," Clinical Neuroradiology, vol. 30, no. 1, pp. 101-107, 2020, Epub 2018 Nov 5. PMID: 30397728. 